\title{
Effect of Different Scented Rice Variety, Planting Geometry and Weed Management Practices on Yield Parameters and Yield at Southern Bastar of Chhattisgarh
}

\author{
Purnima Haldar ${ }^{1}$, Ashwani Kumar Thakur ${ }^{1 *}$, Manish Kumar', \\ G. P. Ayam $^{2}$ and D. P. Singh 3 \\ ${ }^{1}$ Department of Agronomy, ${ }^{2}$ Department of Soil Science and Agricultural Chemistry, \\ ${ }^{3}$ Department of Agricultural Statistics and Social Science, SG College of Agriculture and \\ Research Station, Jagdalpur, IGKV, Raipur (CG) \\ *Corresponding author
}

A B S T R A C T

\begin{tabular}{|l|}
\hline K e y w o r d s \\
Tarunbhog \\
Selection-1, \\
Planting geometry, \\
Weed management, \\
Scented rice
\end{tabular}

A field experiment was conducted at SG College of Agriculture and Research Station, Jagdalpur during Kharif season 2019 to study the effect of different scented rice variety, planting geometry and weed management on yield parameters and yield of rice. The result revealed that variety V2 produced significantly taller plant, more number of tillers, higher dry matter and highest grain yield than the variety V1; in contrast variety V1 produced maximum test weight. Taller plant, more number of tillers, higher rice yield was recorded significantly higher in planting geometry G1 but higher dry matter was recorded in G2 but test weight and harvest index was unable to produce significant effect due to different planting geometry. In case of weed management practice, plant height, number of tillers, plant dry matter, economic yield, straw yield and HI was recorded significantly higher in treatment W1 among all the treatments but test weight was recorded highest in treatment W2.

\section{Introduction}

Paddy is a major food for more than half of the earth's population. Globally, paddy was grown on 161 million hectare, with a yearly production of about 678.7 million tons (FAO, 2016). It is the most important food grain crop of India and is a major source of energy for about 60 per cent of the global population. Asia's food security mainly depends on the irrigated paddy which produces one third of total paddy production. About 90 per cent paddy is produced and consumed in Asian country (Basha et al., 2017). Among the paddy producing countries, India has the largest acreage (43.39 million hectare) and is the second largest producer (104.32 million tons) next to China (207 million tons). The productivity of paddy in India is quite low 2.39 tons $\mathrm{ha}^{-1}$, while the world's average productivity is 4.25 tons $\mathrm{ha}^{-1}$. Chhattisgarh also known paddy bowl of India and it is 
occupies an area of 3.84 million hectare with production and productivity of 6.09 million tons and 1.5 tons $\mathrm{ha}^{-1}$, respectively (Ministry of agriculture \& farmer's welfare, 2016).

Chhattisgarh state is very popular for growing short slender/medium slender aromatic paddy i.e. Dubraj, TulsiManjari, Badshahbhog, Vishnubhog, Gopalbhog, Kubrimohar, Laxmibhog and Jawaphool etc. More than 100 local land races of scented paddy with pleasing aroma are grown in different parts of the Chhattisgarh state. Dubraj is the most popular scented paddy variety in the local markets of Chhattisgarh and with popular brand names "Pride of Chhattisgarh and Chhattisgarh ka Basmati", fetches a high value in the market due to its very good cooking quality, aroma and taste. The spacing and seedlings age are the most important factor for high yield production in aromatic paddy cultivation. Plant geometry affects the normal physiological growth through intraspecific competition (Oad et al., 2001). Wider space allows each plant to produce higher number of tillers but it provides the less number of hills per unit area which results in poor grain yield (Baloch et al., 2002; Gozubenli, 2010 and Kandil et al., 2010).

Weeds are considered one of the major biotic constraints in achieving the higher crop productivity that cause a yield reduction up to 10-90 per cent grain yield (Singh et al., 2004, Kumar et al., 2016d, Kumar et al., 2016a, Kumar et al., 2016b, Kumar et al., 2016c, Chatterjee et al., 2016) . Weeds not only cause vast reduction in yield but also increases the production cost, reduce the input use efficiency, loss of potentially productive lands, loss of range lands and livestock production (Kumar et al., 2015c). Weeds also deplete the nutrients from soil to the tune of 11,3 and $10 \mathrm{~kg} \mathrm{ha}^{-1}$ of NPK (Gautam and Mishra, 1995). Hand weeding is a cultural approach to control weeds is a labour intensive, slow method and very difficult. Among the modern weed management practices, effective weed management through chemical methods plays a vital role in the era of conservation agriculture (Pal et al., 2013, Prakash et al., 2014, Singh et al., 2017).

\section{Materials and Methods}

The experiment was conducted at SG College of Agriculture and Research Station, Jagdalpur (CG) during Kharif season 2019. The experiment was laid out in split - split plot design with three replications. The main plot treatment consists of two scented rice varieties viz. Dubraj selection $-1\left(\mathrm{~V}_{1}\right)$ and Tarunbhog selection-1 $\left(\mathrm{V}_{2}\right)$, sub plot treatments are two planting geometry viz. 20 $\mathrm{cm} \times 15 \mathrm{~cm}\left(\mathrm{G}_{1}\right)$ and $20 \mathrm{~cm} \times 20 \mathrm{~cm}\left(\mathrm{G}_{2}\right)$, and sub- sub plot consists of four weed management practices viz. Pyrazosulfuron Ethyl 10\% WP @ $200 \mathrm{~g} \mathrm{ha}^{-1}$ at 3 DAT + one hand weeding at 3 DAT $\left(\mathrm{W}_{1}\right)$, Bispyribac sodium 10\% SC@ $200 \mathrm{ml} \mathrm{ha}^{-1}$ at $25 \mathrm{DAT}+$ one hand weeding at $50 \mathrm{DAT}\left(\mathrm{W}_{2}\right)$, two hand weeding at 25 and 50 DAT $\left(\mathrm{W}_{3}\right)$ and Absolute control $\left(\mathrm{W}_{4}\right)$. Soil of the experimental site was clay loam (Alfisol) in texture with acidic in nature and medium organic matter and available NPK.

\section{Growth and Yield attributes}

\section{Plant height (cm)}

Plant height is an important growth characteristic that is associated with the productive potential of a plant in relation to biomass and grain yield. The data pertaining to plant height at different stages of crop growth are presented in Table 1, the data reveals that plant height increased progressively with advancement of crop age and reached maximum at harvest. Plant height was recorded significantly taller in variety V2 (Tarunbhog Selection -1) than the variety V1 
(Dubraj Selection -1) during all the growth stages. Plant height is mostly governed by the genetic makeup of the cultivar, but the environmental factors also influence it. Planting geometry, G1 $(20 \mathrm{~cm} \times 15 \mathrm{~cm})$ was observed taller plant than the $\mathrm{G} 2(20 \mathrm{~cm} \times 20$ $\mathrm{cm})$ at 30, 60, 90 DAT and at harvest. The tallest plant height was recorded in closer plant spacing, which might be due to intercompetition among the plants for the interception of maximum solar radiation.

Shah et al., (1991) and Om et al., (1993), who reported that the plant height is significantly affected by the different planting geometry and found maximum plant height in closer spacing. Rasool et al., (2013), who reported that closer plant spacing, produced tallest plant than wider plant spacing in rice. In different weed management practices, treatment W1 (Pyrazosulfuron ethyl $10 \% \mathrm{WP}$ @ $200 \mathrm{~g} \mathrm{ha}^{-1}$ as pre - emergent herbicide + one hand weeding) attained significantly maximum plant height at 60, 90 DAT and at harvest but treatment W3 (Two hand weeding) and W2 (Bispyribac sodium 10\% SC@ $200 \mathrm{ml} \mathrm{ha}^{-1}$ at $25 \mathrm{DAT}+$ one hand weeding at 50 DAT) was found at par during 60 and 90 DAT, and W2 (Bispyribac sodium 10\% SC@200 ml ha ${ }^{-1}$ at 25 DAT+ one hand weeding at 50 DAT) was observed on par at harvest. Non of the treatment was recorded significant due to weed practices at 30 DAT.

This might be due to the better weed control throughout growth stages of rice and better availability of all resources i.e., light, moisture, space and nutrients to rice. As a result of severe competition for resources in uncontrolled weedy crop, the plant height was lower throughout the growth period Ramesha et al., (2017a) and Zahan et al., (2018). Lowest plant height was observed inW4 (Absolute control) during all the growth stages.

\section{Interaction effect on plant height $(\mathrm{cm})$}

Interaction effect on different variety and planting geometry on Plant height are given in 4.2 , the data reveals that interaction effect on Plant height was found significantly affected. Interaction $\mathrm{G} 1 \mathrm{x} \quad \mathrm{V} 2$ and $\mathrm{G} 2 \mathrm{x}$ V2 was recorded significantly higher plant height and rest of the treatment was recorded similar result (Table 2).

\section{Number of tillers (m-2)}

The data pertaining to effective tillers are presented in Table 3. The data reveals that there was significant difference by different treatment during experimental year. Variety V2 (Tarunbhog Selection - 1) recorded significantly more number of tillers per square meter than the variety V1 (Dubraj Selection -1) at 30, 60, 90 DAT and at harvest. Maximum number of tillers was observed at $90 \mathrm{DAT}$, it was due to the favourable condition and stages of rice plant to produce the more tillers. The number of tiller per meter square was decreased and it was not found significantly effect at harvest, it might be due mortality of tillers. In different planting geometry, G1 $(20 \mathrm{~cm} \times 15 \mathrm{~cm})$ recorded significantly maximum number of tillers than the $\mathrm{G} 2(20 \mathrm{~cm} \times 20 \mathrm{~cm})$ during all growth stages. The number of tiller per meter square at 90 DAT and at harvest tillers was decreasing because of closer spacing, competition for utilization of available nutrient, more interception of solar radiation and less inter-plant competition in wider spacing Mahato and Adhikari (2017). This result was also in line with Garcia et al.; (1992), Rodreguze and Ingram (1991); Mohammad et al., (2004), who also observed the maximum number of tillersplant ${ }^{-1}$ in 20 $\mathrm{cm} \times 15 \mathrm{~cm}$ than $20 \mathrm{~cm} \times 20 \mathrm{~cm}$ plant spacing. In different weed management practices, W1 (Pyrazosulfuron ethyl $10 \%$ WP @ $200 \mathrm{~g} \mathrm{ha}^{-1}$ as pre - emergent herbicide + 
one hand weeding) recorded higher number tillers among all the treatment but W3 (Two hand weeding) was found on par with W1 (Pyrazosulfuron ethyl 10\% WP @ $200 \mathrm{~g} \mathrm{ha}^{-1}$ as pre - emergent herbicide + one hand weeding) at all the growth stages and W2 (Bispyribac sodium 10\% SC @ $200 \mathrm{ml} \mathrm{ha}^{-1}$ at 25 DAT+ one hand weeding at 50 DAT) recorded at par at 30 DAT. Negalur et al., (2017),this might be due to reduced crop weed competition during critical growth stages resulted in increased availability of water, nutrients and light. At 90 DAT and at harvest maximum number of tillers evaluated in W1 (Pyrazosulfuron ethyl 10\% WP @ 200 $\mathrm{g} \mathrm{ha}^{-1}$ as pre - emergent herbicide + one hand weeding) followed by W3 (Two hand weeding). It might had decreased bulk density which supported in increased proliferation of root for the uptake of nutrient and soil moisture Singh et al., (2018). Similar view was expressed by Akabar and Ali (2011).

\section{Interaction effect number of tillers $\mathbf{m}-\mathbf{2}$}

Interaction effect on different variety and planting geometry on number of tillers per meter square $^{-2}$ are given in 4.4. The data reveals that interaction effect of planting geometry and variety on number of tillers per meter square was found significantly affected. Interaction at 90 DAT was recorded significantly higher number of tillers per meter square in G1 x V2 and G2 x V1 (Table 4).

\section{Dry matter (g plant $\left.{ }^{-1}\right)$}

The data recorded on plant dry weight of scented rice are presented in Table 5. The data reveals that different varieties recorded significantly effect on dry weight. Plant dry weight was significantly higher in variety V2 (Tarunbhog Selection -1) than the varietyV1 (Dubraj Selection -1) at 30, 60, 90 DAT and at harvest. In different planting geometry, G2 $(20 \mathrm{~cm} \mathrm{x} 20 \mathrm{~cm})$ recorded significantly maximum plant dry matter than the G1 (20 $\mathrm{cm} \times 15 \mathrm{~cm}$ ) at all the growth stages. In case of different weed management practices, W1 (Pyrazosulfuron ethyl $10 \%$ WP @ $200 \mathrm{~g} \mathrm{ha}^{-1}$ as pre - emergent herbicide + one hand weeding) produced significantly higher dry matter among all the treatment at 30, 60, 90 DAT and at harvest, due to least competition with weed. But treatment W2 (Bispyribac sodium 10\% SC @ $200 \mathrm{ml} \mathrm{ha}^{-1}$ at 25 DAT+ one hand weeding at 50 DAT) was found on par at 30 DAT and at harvest.

\section{Results and Discussion}

\section{Test weight (g)}

Test weight of scented rice was affected by different treatment and data are given in Table 6. Scented rice variety, V1 (Dubraj Selection 1) recorded significantly higher than the variety V2 (Tarunbhog selection-1). Different planting geometry treatment did not significantly affect the test weight. In case of different weed management practices, W2 (Bispyribac sodium 10\% SC @ $200 \mathrm{ml} \mathrm{ha}^{-1}$ at 25 DAT+ one hand weeding at 50 DAT) recorded significantly maximum test weight among all the treatment, and lowest test weight was observed in treatment W4 (Absolute control).

\section{Grain yield (q ha $\left.{ }^{-1}\right)$}

Data pertaining to grain yield are presented in Table 6. The data reveals that grain yield of scented rice was not affected significantly by different variety but numerically higher grain yield was observed in variety V2 (Tarunbhog Selection -1) than the variety V1 (Dubraj selection-1). In different planting geometry, treatment $\mathrm{G} 1(20 \mathrm{~cm} \times 15 \mathrm{~cm})$ was produced higher grain yield than the G2 (20 cm x 20 $\mathrm{cm})$, it was because of greater weed suppression, which resulted in higher crop dry matter accumulation, more effective tillers 
and less yield due to less dry matter accumulation, and less number of tillers per meter square. Khalil et al., (2016), Ghuman et al., (2008) and Kaur and Singh (2014). In case of different weed management practices, W1 (Pyrazosulfuron ethyl 10\% WP @ $200 \mathrm{~g}$ a.i. $\mathrm{ha}^{-1}+$ one hand weeding) recorded significantly higher grain yield but it was found on par with W3 (Two hand weeding at 25 and 50 DAT) and W2 (Bispyribac sodium 10\% SC @ $200 \mathrm{ml} \mathrm{ha}^{-1}$ at $25 \mathrm{DAT}+$ one hand weeding at $50 \mathrm{DAT}$ ).Due to minimum crop and weed competition and less number of weeds, increased soil moisture and nutrient availability resulting superior production factor like affective tillers, panicle length, grain panicle ${ }^{-1}$ which cumulatively contributed to higher grain yield, Ramesha $e t$ al., (2017a) and Ghosh et al., (2016).

\section{Straw yield (q ha $\left.{ }^{-1}\right)$}

Data presented in Table 6, reveals that straw yield was not affected significantly by different variety and planting geometry but straw yield was significantly superior in W2 (Bispyribac sodium 10\% SC @ $200 \mathrm{ml} \mathrm{ha}^{-1}$ at $25 \mathrm{DAT}+$ one hand weeding at $50 \mathrm{DAT}$ ) and it was recorded on par with W3 (Two hand weeding at 25 and 50 DAT) in different weed management practices. Similar result was also observed by Saju et al., (2019).

Table.1 Plant height of rice as influence by different variety, planting geometry and weed management practices

\begin{tabular}{|c|c|c|c|c|}
\hline \multirow[t]{2}{*}{ Treatment } & \multicolumn{4}{|c|}{ Plant height (cm) } \\
\hline & At 30 DAT & At 60 DAT & At 90 DAT & At harvest \\
\hline & \multicolumn{4}{|l|}{ Variety } \\
\hline V1 & 54.01 & 87.97 & 132.85 & 137.17 \\
\hline V2 & 60.38 & 92.84 & 137.56 & 140.67 \\
\hline SEm \pm & 1.04 & 0.78 & 0.33 & 0.40 \\
\hline \multirow[t]{2}{*}{ CD at 0.05} & 6.40 & 1.14 & 2.10 & 1.43 \\
\hline & \multicolumn{4}{|l|}{ Geometry } \\
\hline G1 & 59.02 & 91.59 & 136.98 & 140.62 \\
\hline G2 & 55.37 & 89.23 & 133.43 & 137.21 \\
\hline SEm \pm & 0.81 & 0.29 & 0.47 & 0.41 \\
\hline \multirow[t]{2}{*}{$C D$ at 0.05} & 3.18 & 1.14 & 1.84 & 1.46 \\
\hline & \multicolumn{4}{|c|}{ Weed management } \\
\hline W1 & 59.50 & 92.05 & 137.06 & 140.74 \\
\hline W2 & 56.32 & 91.23 & 134.79 & 138.62 \\
\hline W3 & 57.99 & 91.23 & 136.94 & 140.53 \\
\hline W4 & 54.98 & 87.25 & 132.03 & 135.79 \\
\hline $\begin{array}{l}\text { SEm } \pm \\
\text { CD at } 0.05\end{array}$ & $\begin{array}{l}1.50 \\
\text { NS }\end{array}$ & $\begin{array}{l}1.04 \\
3.03\end{array}$ & $\begin{array}{l}1.01 \\
2.94\end{array}$ & $\begin{array}{l}0.41 \\
0.21\end{array}$ \\
\hline
\end{tabular}

V1-Dubraj Selection-1, V2- Tarunbhog Selection-1, G1- $20 \mathrm{~cm}$ x $15 \mathrm{~cm}, \mathrm{G} 2-20 \mathrm{~cm}$ x $20 \mathrm{~cm}$, W1- Pyrazosulfuron ethyl 10\% WP + One hand weeding, W2- Bispyribac Sodiun 10\% SC +One hand weeding, W3- Two Hand Weeding and W4-Absolute control 
Table.2 Interaction effect on plant height of rice as influence by different variety, planting geometry and weed management practices

\begin{tabular}{|c|c|c|c|c|c|c|c|}
\hline \multicolumn{8}{|c|}{ Plant height $(\mathrm{cm})$ at 90 DAT } \\
\hline \multirow[t]{3}{*}{ Treatment } & \multicolumn{6}{|c|}{ Variety } & \multirow[t]{3}{*}{ Mean } \\
\hline & \multicolumn{3}{|c|}{ V1 } & \multicolumn{3}{|c|}{ V2 } & \\
\hline & \multicolumn{6}{|c|}{ Geometry } & \\
\hline Weed management & G1 & G2 & \multicolumn{2}{|c|}{ G1 } & \multicolumn{2}{|c|}{ G2 } & \\
\hline W1 & 136.05 & 132.30 & \multicolumn{2}{|c|}{141.77} & \multicolumn{2}{|c|}{138.10} & 137.06 \\
\hline W2 & 133.90 & 130.33 & \multicolumn{2}{|c|}{139.03} & \multicolumn{2}{|l|}{135.90} & 134.79 \\
\hline W3 & 137.480 & 133.17 & \multicolumn{2}{|c|}{140.27} & \multicolumn{2}{|l|}{136.83} & 136.94 \\
\hline W4 & 131.23 & 128.33 & \multicolumn{2}{|c|}{136.10} & \multicolumn{2}{|l|}{132.45} & 132.03 \\
\hline Mean V & \multicolumn{2}{|l|}{132.85} & \multicolumn{4}{|c|}{137.56} & \\
\hline \multirow[t]{2}{*}{ Mean G } & \multicolumn{2}{|l|}{136.98} & \multicolumn{4}{|c|}{133.43} & \\
\hline & V & G & W & $\mathbf{V} \times \mathbf{G}$ & $\mathbf{V} \times \mathbf{W}$ & $\mathbf{G} \times \mathbf{W}$ & V x G x W \\
\hline SEM $m \pm$ & 0.60 & 0.56 & 1.01 & 0.56 & 1.43 & 1.43 & 2.02 \\
\hline$C D$ at 0.05 & 3.71 & 2.20 & 2.95 & 2.20 & NS & NS & NS \\
\hline
\end{tabular}

V1-Dubraj Selection-1, V2- Tarunbhog Selection-1, G1- $20 \mathrm{~cm}$ x $15 \mathrm{~cm}, \mathrm{G} 2-20 \mathrm{~cm}$ x $20 \mathrm{~cm}$, W1- Pyrazosulfuron ethyl 10\% WP + One hand weeding, W2- Bispyribac Sodiun 10\% SC +One hand weeding, W3- Two Hand Weeding and W4-Absolute control

Table.3 Number of tillers $\mathrm{m}^{-2}$ of rice as influence by different variety, planting geometry and weed management practices

\begin{tabular}{|c|c|c|c|c|}
\hline \multirow[t]{2}{*}{ Treatment } & \multicolumn{4}{|c|}{ Number of tillers $\mathbf{m}^{-2}$} \\
\hline & At 30DAT & At 60 DAT & At 90 DAT & At harvest \\
\hline & \multicolumn{4}{|c|}{ Variety } \\
\hline V1 & 224.81 & 269.12 & 307.93 & 303.25 \\
\hline V2 & 262.10 & 305.78 & 342.65 & 337.65 \\
\hline SEm \pm & 8.79 & 9.47 & 7.25 & 8.30 \\
\hline \multirow[t]{2}{*}{$C D$ at 0.05} & NS & NS & NS & NS \\
\hline & \multicolumn{4}{|c|}{ Geometry } \\
\hline G1 & 267.33 & 320.11 & 364.13 & 358.41 \\
\hline G2 & 219.58 & 254.79 & 286.46 & 282.19 \\
\hline SEm \pm & 4.89 & 4.76 & 5.76 & 6.87 \\
\hline \multirow[t]{2}{*}{$C D$ at 0.05} & 19.08 & 18.57 & 22.49 & 26.82 \\
\hline & \multicolumn{4}{|c|}{ Weed management } \\
\hline W1 & 265.26 & 324.15 & 366.79 & 357.41 \\
\hline W2 & 242.21 & 286.51 & 326.93 & 325.19 \\
\hline W3 & 257.49 & 304.29 & 345.26 & 341.72 \\
\hline W4 & 208.88 & 234.85 & 262.21 & 257.49 \\
\hline $\operatorname{SEm} \pm$ & 9.41 & 8.99 & 9.48 & 10.40 \\
\hline$C D$ at 0.05 & 27.50 & 26.24 & 27.69 & 30.37 \\
\hline
\end{tabular}

V1-Dubraj Selection-1, V2- Tarunbhog Selection-1, G1- $20 \mathrm{~cm}$ x $15 \mathrm{~cm}, \mathrm{G} 2-20 \mathrm{~cm}$ x $20 \mathrm{~cm}$, W1- Pyrazosulfuron ethyl 10\% WP + One hand weeding, W2- Bispyribac Sodiun 10\% SC +One hand weeding, W3- Two Hand Weeding and W4-Absolute control 
Table.4 Interaction effect on number of tillers $\mathrm{m}^{-2}$ at 90 DATof rice as influence by different variety, planting geometry and weed management practices

\begin{tabular}{|c|c|c|c|c|c|c|c|}
\hline \multicolumn{8}{|c|}{ Number of tillers $\mathrm{m}^{-2}$ at 90 DAT } \\
\hline \multirow[t]{3}{*}{ Treatment } & \multicolumn{6}{|c|}{ Variety } & \multirow[t]{3}{*}{ Mean } \\
\hline & \multicolumn{3}{|c|}{ V1 } & \multicolumn{3}{|c|}{$\mathbf{V 2}$} & \\
\hline & \multicolumn{6}{|c|}{ Geometry } & \\
\hline Weedmanagement & \multicolumn{3}{|c|}{ G2 } & G1 & \multicolumn{2}{|c|}{ G2 } & \\
\hline W1 & \multicolumn{2}{|l|}{374.41} & 333.33 & 437.73 & \multicolumn{2}{|c|}{321.67} & 366.79 \\
\hline W2 & \multicolumn{2}{|l|}{333.30} & 287.50 & 397.74 & \multicolumn{2}{|c|}{289.17} & 326.93 \\
\hline W3 & \multicolumn{2}{|l|}{358.86} & 309.17 & 425.51 & \multicolumn{2}{|c|}{287.50} & 345.26 \\
\hline W4 & 247.75 & & 219.17 & 337.75 & \multicolumn{2}{|c|}{244.17} & 262.21 \\
\hline Mean V & \multicolumn{3}{|c|}{307.94} & \multicolumn{3}{|c|}{342.65} & \\
\hline \multirow[t]{2}{*}{ Mean G } & \multicolumn{3}{|c|}{286.46} & \multicolumn{3}{|c|}{286.46} & \\
\hline & $\mathbf{V}$ & G & $\mathbf{W}$ & $\mathbf{V} \times \mathbf{G}$ & $\mathbf{V} \times \mathbf{W}$ & $\mathbf{G} \times \mathbf{W}$ & $\mathbf{V} \times \mathbf{G} \times \mathbf{W}$ \\
\hline SEM m \pm & 7.25 & 5.76 & 9.48 & 8.15 & 13.41 & 13.41 & 18.97 \\
\hline$C D$ at 0.05 & NS & 22.49 & 27.69 & 31.80 & NS & NS & NS \\
\hline
\end{tabular}

V1-Dubraj Selection-1, V2- Tarunbhog Selection-1, G1- $20 \mathrm{~cm}$ x $15 \mathrm{~cm}, \mathrm{G} 2-20 \mathrm{~cm}$ x $20 \mathrm{~cm}$, W1- Pyrazosulfuron ethyl 10\% WP + One hand weeding, W2- Bispyribac Sodiun 10\% SC +One hand weeding, W3- Two Hand Weeding and W4-Absolute control

Table.5 Dry weight plant $^{-1}$ of rice as influence by different variety, planting geometry and weed management practices

\begin{tabular}{|c|c|c|c|c|}
\hline \multirow[t]{2}{*}{ Treatment } & \multicolumn{4}{|c|}{ Dry weight plant $^{-1}(\mathrm{~g})$} \\
\hline & At 30 DAT & At 60 DAT & At 90 DAT & At harvest \\
\hline & \multicolumn{4}{|c|}{ Variety } \\
\hline V1 & 3.28 & 22.61 & 55.07 & 63.16 \\
\hline $\mathbf{V 2}$ & 3.94 & 24.61 & 59.42 & 67.57 \\
\hline SEm \pm & 0.11 & 0.22 & 0.61 & 0.70 \\
\hline \multirow[t]{2}{*}{$C D$ at 0.05} & 0.65 & 1.36 & 3.76 & 4.31 \\
\hline & \multicolumn{4}{|c|}{ Geometry } \\
\hline G1 & 3.26 & 22.32 & 54.92 & 63.39 \\
\hline G2 & 3.96 & 24.90 & 59.57 & 67.35 \\
\hline SEm \pm & 0.08 & 0.26 & 0.30 & 0.42 \\
\hline \multirow[t]{2}{*}{$C D$ at 0.05} & 0.33 & 1.01 & 1.17 & 1.63 \\
\hline & \multicolumn{4}{|c|}{ Weed management } \\
\hline W1 & 4.30 & 26.25 & 61.88 & 68.17 \\
\hline W2 & 3.43 & 23.09 & 56.21 & 64.78 \\
\hline W3 & 3.90 & 24.13 & 57.71 & 67.07 \\
\hline W4 & 2.81 & 20.96 & 53.17 & 61.43 \\
\hline SEm \pm & 0.15 & 0.56 & 0.65 & 0.82 \\
\hline$C D$ at 0.05 & 0.44 & 1.70 & 1.89 & 2.40 \\
\hline
\end{tabular}

V1-Dubraj Selection-1, V2- Tarunbhog Selection-1, G1- $20 \mathrm{~cm}$ x $15 \mathrm{~cm}$, G2-20 cm x $20 \mathrm{~cm}$, W1- Pyrazosulfuron ethyl 10\% WP + One hand weeding, W2- Bispyribac Sodiun 10\% SC +One hand weeding, W3- Two Hand Weeding and W4-Absolute control 
Table.6 Test weight (g), Grain yield, Straw yield and Harvest Indexof rice as influence by different variety, planting geometry and weed management practices

\begin{tabular}{|c|c|c|c|c|}
\hline Treatment & Test weight (g) & $\begin{array}{c}\text { Grain yield } \\
\left(\mathbf{q} \text { ha }^{-1}\right)\end{array}$ & $\begin{array}{c}\text { Straw yield } \\
\left(\mathbf{q} \mathbf{h a}^{-1}\right)\end{array}$ & Harvest Index (\%) \\
\hline \multicolumn{5}{|c|}{ Variety } \\
\hline V1 & 18.28 & 41.28 & 54.46 & 42.90 \\
\hline V2 & 14.43 & 43.93 & 56.73 & 43.54 \\
\hline SEm \pm & 0.05 & 0.64 & 0.82 & 0.03 \\
\hline$C D$ at 0.05 & 0.29 & NS & $\mathrm{NS}$ & NS \\
\hline \multicolumn{5}{|c|}{ Geometry } \\
\hline G1 & 16.33 & 43.90 & 56.35 & 43.59 \\
\hline G2 & 16.38 & 41.31 & 54.84 & 42.85 \\
\hline SEm \pm & 0.14 & 0.61 & 0.75 & 0.31 \\
\hline$C D$ at 0.05 & NS & 2.37 & NS & NS \\
\hline \multicolumn{5}{|c|}{ Weed management } \\
\hline W1 & 16.48 & 46.08 & 53.88 & 46.03 \\
\hline W2 & 16.80 & 44.17 & 60.94 & 41.92 \\
\hline W3 & 16.63 & 45.07 & 56.74 & 44.20 \\
\hline W4 & 15.49 & 35.10 & 50.83 & 40.74 \\
\hline SEMm \pm & 0.16 & 0.71 & 1.06 & 0.29 \\
\hline$C D$ at 0.05 & 0.48 & 2.92 & 2.53 & 0.85 \\
\hline
\end{tabular}

V1-Dubraj Selection-1, V2- Tarunbhog Selection-1, G1- $20 \mathrm{~cm}$ x $15 \mathrm{~cm}, \mathrm{G} 2-20 \mathrm{~cm}$ x $20 \mathrm{~cm}$, W1- Pyrazosulfuron ethyl 10\% WP + One hand weeding, W2- BispyribacSodiun 10\% SC +One hand weeding, W3- Two Hand Weeding and W4-Absolute control

Table.7 Interaction effect on grain yield $\left(\mathrm{q} \mathrm{ha}^{-1}\right)$ of rice as influence by different variety, planting geometry and weed management practices

\begin{tabular}{|c|c|c|c|c|c|c|c|}
\hline \multicolumn{8}{|c|}{ Grain yield $\left(q\right.$ ha $\left.^{-1}\right)$} \\
\hline \multirow[t]{3}{*}{ Treatment } & \multicolumn{6}{|c|}{ Variety } & \multirow[t]{3}{*}{ Mean } \\
\hline & \multicolumn{3}{|c|}{ V1 } & \multicolumn{3}{|c|}{$\mathbf{V} 2$} & \\
\hline & \multicolumn{6}{|c|}{ Geometry } & \\
\hline $\begin{array}{c}\text { Weed } \\
\text { management }\end{array}$ & G1 & \multicolumn{2}{|r|}{ G2 } & G1 & \multicolumn{2}{|c|}{ G2 } & \\
\hline W1 & 48.00 & \multicolumn{2}{|r|}{44.93} & 47.47 & \multicolumn{2}{|c|}{43.93} & 46.08 \\
\hline W2 & 45.33 & \multicolumn{2}{|r|}{40.00} & 46.40 & \multicolumn{2}{|c|}{44.93} & 44.17 \\
\hline W3 & 45.47 & \multicolumn{2}{|r|}{43.07} & 47.07 & \multicolumn{2}{|c|}{44.67} & 45.07 \\
\hline W4 & 35.07 & \multicolumn{2}{|r|}{28.40} & 36.40 & \multicolumn{2}{|c|}{40.53} & 35.10 \\
\hline Mean V & \multicolumn{3}{|c|}{41.28} & \multicolumn{3}{|c|}{43.93} & \\
\hline \multirow[t]{2}{*}{ Mean G } & \multicolumn{3}{|c|}{43.90} & \multicolumn{3}{|c|}{41.31} & \\
\hline & $\mathbf{V}$ & $\mathbf{G}$ & $\mathbf{W}$ & $\mathbf{V} \times \mathbf{G}$ & $\mathbf{V} \times \mathbf{W}$ & $\mathbf{G} \times \mathbf{W}$ & $\mathbf{V} \times \mathbf{G} \times \mathbf{W}$ \\
\hline SEM m \pm & 0.64 & 0.61 & 0.71 & 0.86 & 1.00 & 1.00 & 1.42 \\
\hline$C D$ at 0.05 & NS & 2.37 & 2.07 & NS & 2.92 & NS & 4.13 \\
\hline
\end{tabular}

V1-Dubraj Selection-1, V2- Tarunbhog Selection-1, G1- $20 \mathrm{~cm}$ x $15 \mathrm{~cm}$, G2-20 cm x $20 \mathrm{~cm}$, W1- Pyrazosulfuron ethyl 10\% WP + One hand weeding, W2- Bispyribac Sodiun 10\% SC +One hand weeding, W3- Two Hand Weeding and W4-Absolute control 
Table.8 Interaction effect on harvest index (\%) of rice as influence by different variety, planting geometry and weed management practices

\begin{tabular}{|c|c|c|c|c|c|c|c|}
\hline \multicolumn{8}{|c|}{ Harvest index (\%) } \\
\hline \multirow[t]{3}{*}{ Treatment } & \multicolumn{6}{|c|}{ Variety } & \multirow[t]{3}{*}{ Mean } \\
\hline & \multicolumn{3}{|c|}{ V1 } & \multicolumn{3}{|c|}{$\mathbf{V} 2$} & \\
\hline & \multicolumn{6}{|c|}{ Geometry } & \\
\hline Weed management & \multicolumn{3}{|r|}{ G2 } & G1 & \multicolumn{2}{|r|}{ G2 } & \\
\hline W1 & 45.52 & \multicolumn{2}{|r|}{46.17} & 46.90 & \multicolumn{2}{|r|}{45.52} & 46.03 \\
\hline $\mathbf{W 2}$ & 42.37 & \multicolumn{2}{|r|}{40.04} & 43.53 & \multicolumn{2}{|r|}{41.72} & 41.92 \\
\hline W3 & 45.52 & \multicolumn{2}{|r|}{42.88} & 44.26 & \multicolumn{2}{|r|}{44.14} & 44.20 \\
\hline W4 & 40.04 & & 40.65 & 40.60 & \multicolumn{2}{|c|}{41.67} & 40.74 \\
\hline Mean V & \multicolumn{3}{|c|}{42.90} & \multicolumn{3}{|c|}{43.54} & \\
\hline \multirow[t]{2}{*}{ Mean G } & \multicolumn{3}{|c|}{43.59} & \multicolumn{3}{|c|}{42.85} & \\
\hline & $\mathbf{V}$ & $\mathbf{G}$ & $\mathbf{W}$ & $\mathbf{V} \times \mathbf{G}$ & $\mathbf{V} \times \mathbf{W}$ & $\mathbf{G} \times \mathbf{W}$ & $\mathbf{V} \times \mathbf{G} \times \mathbf{W}$ \\
\hline SEM m \pm & 0.23 & 0.31 & 0.29 & 0.44 & 0.41 & 0.41 & 0.59 \\
\hline$C D$ at 0.05 & NS & NS & 0.85 & NS & NS & 1.21 & NS \\
\hline
\end{tabular}

V1-Dubraj Selection-1, V2- Tarunbhog Selection-1, G1- $20 \mathrm{~cm}$ x $15 \mathrm{~cm}$, G2-20 cm x $20 \mathrm{~cm}$, W1- Pyrazosulfuron ethyl 10\% WP + One hand weeding, W2- Bispyribac Sodiun 10\% SC +One hand weeding, W3- Two Hand Weeding and W4-Absolute control

\section{Harvest index (\%)}

Data presented in Table 6, reveals that harvest index was not affected significantly by different scented varieties but it was recorded significantly superior in G1 $(20 \mathrm{~cm} \times 15 \mathrm{~cm})$ over the G2 $(20 \mathrm{~cm} \times 20 \mathrm{~cm})$. It might be the resultant effects of highest tillers hill ${ }^{-1}$ and grains panicle ${ }^{-1}$. In case of different weed management practices, W1 (Pyrazosulfuron ethyl 10 \% WP @ 200 g a.i. ha ${ }^{-1}+$ one hand weeding) recorded significantly higher harvest index among all the treatment but it was found at par with W3 (Two hand weeding). The herbicide application had similar effect to hand weeding regarding the weed control in scented rice, might be attributed to long term effective control of weeds during the growing period of crop Hasanuzzaman et al., (2008).

\section{Interaction effect on grain yield $\left(q\right.$ ha $\left.^{-1}\right)$}

Interaction effect on different varieties, planting geometry and weed management practices on grain yield are given in Table 7 , the data reveals that interaction effect of varieties $\mathrm{x}$ planting geometry $\mathrm{x}$ different weed management practices, were found significantly. The interaction table reveals that V1 x G1 x W1 was recorded significantly higher grain yield and which was found on par with V1 x G1 x W2 and W3. Variety V1 x G2 x W1 was recorded significantly higher grain yield and it was found on par with V1 x G2 x W3. Variety V2 x G1 x W1 recorded significantly higher economic yield and which was recorded statistically on par with V2 $\mathrm{x}$ G1 x W3 and W1, while V2 x G2 x W was produced significantly similar result in interaction.

\section{Interaction effect on Harvest index (\%)}

Interaction effect on different planting geometry and weed management practices on $\mathrm{HI}$ are given in Table 8 , the data reveals that interaction effect of harvest index was found significantly higher with G1 x W1 and W3 but G2 observed higher HI with W4. 


\section{References}

Akabar. N., Ali, E. 2011. Weed management improves yield and quality of direct seeded rice. Australian Journal of Crop Science, 5(6): 688-694.

Baloch, A. W., Soomro, A. M., Javed, M. A. and Ahmed, M. 2002. Optimum plant density for high yield in rice. Asian Journal Plant Science, 1: 25-27.

Basha, J., Venkatesh, B. and Kumar, S. 2017. Rice (Oryzasativa L.) response to Planting Geometry and Method of Planting. International Journal of New Technologies in Science and Engineering, 4(1): 13-21.

Chatterjee, D., Kumar, R., Kuotsu, R. and Deka, B.C. 2016. Validation of traditional weed control method through common salt application in the hill region of Nagaland. Current Science, 110(8): 1159-1167.

FAO, 2016.Food and Agriculture Organization, FAOSTAT Database FAO, Rome, www.faostat.fao.org.

Garcia, F.D., Escabarte, R. S. J. R., Sta Cruz, P. C. and Obein. S. R. 1992. Improving the yield potential of lowland rice varieties through appropriate plant spacing and nitrogen management. Philippine J Crop Sci 17(1): 26.

Gautam, K. C. and Mishra, J. S. 1995. Problem, prospects and new approaches in weed management. Pest Information, 21:7-19.

Ghosh, D., Singh, U. P., Ray, K. and Das, A. 2016. Weed management through herbicide application in direct-seeded rice and yield modelling by artificial neural network. Spanish Journal of Agricultural Research, 14(2): 21719292.

Ghuman, R. S., Brar, L. S. and Walia, U. S. 2008. Role of Variety and Plant Geometry on Weed Management in Transplanted Rice (Oryza sativa L.). Indian Journal Weed Science, 40 (3 \& 4) : 137-141.

Gozubenli, H. 2010. Influence of planting pattern and plant density on the performance of maize hybrids in the eastern Mediterranean condition. Int. J. Agric. Biol., 12: 556-560.

Hasanuzzaman, M., Islam, Md, Obaidul. and Bapari, Md. S. 2008. Efficacy of different herbicides over manual weeding in controlling weeds in transplanted rice. Australian Journal of Crop Science, 2(1): 18-24.

Kandil, A. A., El-Kalla, S. E., Badawi, A. T. and El-Shayb, O. M. 2010. Effect of hill spacing, nitrogen levels and harvest date on rice productivity and grain quality. Crop Environ., 1: 22-26.

Kaur, S. and Singh. 2014. Influence of crop density on weeds, growth and yield of direct-seeded rice. Indian Journal of Weed Science, 46(4): 318-321.

Khalil, M. E., Kaium, C., Sabagh, A. EL., Barutcular, C. and Islam, M. S. 2016. Effect of planting geometry on yield and yield attributes of aromatic rice genotypes. Agricultural Advances, 5(9): 349-357.

MAFW, 2016.Agricultural Statistics at a glance 2016. Directorate of Economics and Statistics, Ministry of Agriculture and Farmers Welfare, GOI,

Mahato, M. and Adhikari, B. B. 2017. Effect of Planting Geometry on Growth of Rice Varieties. Int. J. Appl. Sci. Biotechnol, 5(4): 423-429.

Mohammad, A., Khan, M. A., Khan, E. J. and Muhammad, R. 2004. Effect of increased plant density and fertilizer dose on the yield of rice variety IR6.Faculty of Agriculture, Gomal University, Dera Ismail Khan.

Negalur, R. B., Ananda, N., Guruprasad, G.S. and Narappa, G. 2017. New herbicide molecule combination for control of complex weed flora in transplanted rice (Oryza sativa L.). International Journal of Chemical Studies, 5(4): 1592-1597.

Oad, F. C., Solangi, B. K., Samo, M. A., Lakho, A. A., Hassan, Z. U. and Oad, N. L. 2001. Growth, yield and relationship of rape seed under different row spacing. 
Int. J. Agric. Biol., 3: 475-476.

Om, H., Singh, O. P. and Joon, R. K. 1993. Effect of time of transplanting and spacing on Basmati rice. Haryana J. Agron., 9(1): 87.

Pal, V., Singh, M. M., Kumar, R. and Verma S. S. 2013. Response of irrigation scheduling and integrated nutrition on scented rice (Oryza sativa L.). Bioinfolet, 10(4C):1528-1530.

Prakash, C., Koli, N.R., Shivran, R.K., Sharma, J.C., Kumar, R. 2014. Response of nitrogen levels and weed management practices on productivity of rice (Oryza sativa L.) under aerobic condition. Bioinfolet, 11(1A): 145-148.

Ramesha, Y. M., Ajaya kumar, M. Y., Manjunathabhanuvally, Murthy, D. K. and Roopashree, D. H. 2017a. BioEfficacy of Pyrazosulfuron Ethyl 10\% WP against Weeds in Transplanted Rice. Acta Scientific Agriculture, 1 (1): 06-10.

Rasool, F., Habib, R. and Bhat, M. I. 2013.Agronomic evaluation of rice (Oryza sativa L.) for plant spacing and seedlings per hill under temperate condition. African Journal of Agricultural Research, 8(37): 46504653.

Rodreguez, R. C. and Ingram, K.T. 1991. Establishment and yield of rainfed lowland dry seeded rice as influenced by row spacing. Philippine J Crop Sci., 16(1): S10.

Saju, S. M., Thavaprakaash, N., Sakthivel, N. and Malathi, P. 2019. Influence of high density planting on growth and yield of rice (Oryza sativa L.) under modified system of rice intensification. Journal of Pharmacognosy and Phytochemistry, 8(3): 3376-3380.

Shah, M. H., Khushu, M. K., Khandey, B. A. and Bali, A. S. 1991. Effect of spacing and seedling per hill on transplanted rice under late sown conditions. Indian J. Agron., 36(2): 274-275.

Singh, G. P., Roy, D. K and Yadav, S. 2018. Effect of herbicides combinations and hand weeding on growth, yield and weed population in transplanted rice (Oryzasativa L.). International Journal of Chemical Study, 6(5): 154-157.

Singh, S. K., Abraham, T., Kumar, R. and Kumar, R. 2017. Response of crop establishment methods and split application of nitrogen on productivity of rice under irrigated ecosystem. Environment and Ecology, 35(2A): 859862.

Singh, U.P., Singh, Y. and Kumar, V. 2004. Effect of weed management and cultivars on boro rice and weeds. Indian Journal of Weed Science, 36: 57-59.

Zahan, T., Hashem, A., Rahman,M., Bell, R.W. and Begum. M. 2018. Efficacy of Herbicides in Non-Puddled Transplanted Rice under Conservation Agriculture Systems and Their Effect on Establishment of the Succeeding Crops. Acta Scientifica Malaysia, 2(1): 17-25.

\section{How to cite this article:}

Purnima Haldar, Ashwani Kumar Thakur, Manish Kumar, G. P. Ayam and Singh, D. P. 2021. Effect of Different Scented Rice Variety, Planting Geometry and Weed Management Practices on Yield Parameters and Yield at Southern Bastar of Chhattisgarh. Int.J.Curr.Microbiol.App.Sci. 10(01): 102-112. doi: https://doi.org/10.20546/ijcmas.2021.1001.012 